\title{
Correlation effects in the optical spectra of porphyrin oligomer chains: Exciton confinement and length dependence
}

\author{
Conor Hogan, ${ }^{1,2}$ Maurizia Palummo, ${ }^{2}$ Johannes Gierschner, ${ }^{3}$ and Angel Rubio ${ }^{4}$ \\ ${ }^{1}$ Consiglio Nazionale delle Ricerche, Istituto di Struttura della Materia (CNR-ISM), via Fosso del Cavaliere \\ 100, 00133 Rome, Italy \\ ${ }^{2}$ Department of Physics, CNISM and European Theoretical Spectroscopy Facility (ETSF), University of Rome \\ "Tor Vergata," Via della Ricerca Scientifica 1, 00133 Roma, Italy \\ ${ }^{3}$ Madrid Institute for Advanced Studies, IMDEA in Nanoscience, C/Faraday 9, Ciudad Universitaria de \\ Cantoblanco, 28049 Madrid, Spain \\ ${ }^{4}$ Nano-Bio Spectroscopy Group and ETSF, Dpto. de Física de Materiales, Universidad del País Vasco \\ UPV/EHU and Centro Mixto CSIC-UPV/EHU, Av. Tolosa 72, E-20018 San Sebastián, Spain
}

(Received 11 October 2012; accepted 13 December 2012; published online 10 January 2013)

\begin{abstract}
Excited states of ethylene-linked free-base porphyrin oligomers and polymer are studied using manybody perturbation theory (MBPT) within the $G W$ approximation and the Bethe-Salpeter equation. Trends in the electronic levels with oligomer length are analysed and the correct long-range behaviour in the band gap is obtained. High polarizabilities and strong redshifts in the optical absorption peaks are predicted in agreement with observations on other strongly conjugated oligoporphyrins. We explain these trends by means of spatial and spectral analyses of the exciton character. Although Wannier-Mott and charge-transfer excitons are identified in the optical spectra, the strongest polarizabilities are actually associated with small, tightly bound excitons (Frenkel-like), in contrast to expectations. Furthermore, the common procedure of extrapolating polymer properties from oligomer calculations is examined from a MBPT perspective. (c) 2013 American Institute of Physics. [http://dx.doi.org/10.1063/1.4773582]
\end{abstract}

\section{INTRODUCTION}

Porphyrin oligomers have attracted widespread interest recently due to their extraordinary optical and electronic properties, which makes them suitable for a wide variety of applications, including biosensing, molecular switches and wires, and optoelectronics. ${ }^{1-4}$ The main characteristics of these oligomers, which occur as a direct consequence of $\pi$-conjugation, are high polarizabilities, enhanced oscillator strengths, and low-lying electronic transitions, and as such they offer great potential for building highly efficient organic solar cell devices. ${ }^{5,6}$ In the field of nonlinear optical applications their performance already exceeds that of many organic molecules, ${ }^{7,8}$ while the high charge carrier mobilities manifested in highly conjugated porphyrin derivatives have lead to reports of greatly improved performances in organic field effect transistors (OFETs). ${ }^{9}{ }^{10}$ Hence there is much interest in a thorough optical and electronic characterization of porphyrin oligomers and in understanding how to tune their structural motifs (such as the chain length, bridging unit, central metal atom, and so on) in order to optimize the desired functionality.

Synthesis of $\pi$-conjugated porphyrin oligomers ${ }^{4}$ has been achieved by covalently linking porphyrins with alkynes, ${ }^{11-14}$ alkenes, ${ }^{15-18}$ and edge-fused aromatic units. ${ }^{19,20}$ The observed change in polarizability with chain length is determined by the type and localization of bound electron-hole pairs (excitons) within the oligomers, as dictated by the nature of the coupling between each porphyrin unit. ${ }^{21}$ This coupling depends on the alignment of the adjacent planar macrocycles as well as the structure and relative alignment of the molecular bridge. For instance, in Zn-porphyrin oligomers linked by aromatic units oriented perpendicular to the macrocyclic plane, only a weak splitting is observed in the most intense (B, or Soret) peak of the absorption spectrum with respect to that of the monomer. Following Kasha, ${ }^{21}$ this is understood to derive from weak electrostatic interactions between the transition dipole moments of the different macrocyclic units, and is indicative of strongly bound, localized excitons. ${ }^{22}$ In contrast, the optical absorption of butadiynylene-bridged oligomers is characterized by strong spectral redshifts and significant increases in intensity. ${ }^{22}$ Such behaviour is interpreted as deriving from the electronic coupling between porphyrin units giving rise to more weakly bound, delocalized excitons. These two extremes are often classified as Frenkel and WannierMott excitons, ${ }^{23,24}$ respectively, although in reality there is considerable overlap between them. Controlling the delocalization and dissociation properties of these excitons by changing the oligomer length or molecular bridge is of prime importance in optimizing the performance of organic solid cell devices.

Computational approaches to clarify the excitonic features as a function of the chain length are thus of fundamental importance. Up to now, there have been several theoretical studies of singlet excited states of oligoporphyrins, mostly focusing on those containing alkynyl bridges. ${ }^{25-27}$ In this paper we provide a systematic analysis of the optical excitations in meso-meso ethylene-linked porphyrin oligomer chains by means of ab initio calculations of the electronic states, optical spectra, and exciton distribution. Alkene-linked porphyrin dimers have been successfully synthesized and their spectra measured. ${ }^{15,16,18}$ This choice of bridge allows the chromophores to remain close (meso carbons $\leq 4 \AA$ apart) and 
coplanar without having to rotate due to steric interactions: instead, the macrocycles adopt a small vertical stacking, ensuring that good overlap of $\pi$-electron clouds is maintained while minimising $\mathrm{H}-\mathrm{H}$ repulsion across the bridge. As the systems to be studied range from isolated molecules and oligomers through to infinite polymer chains, it is necessary to use a theoretical framework which can adequately treat long and short range exchange and correlation effects on an equal and consistent basis, and which is technically feasible. Several approaches are possible. Time-dependent density functional theory (TDDFT) has been widely used to predict and obtain the optical spectra of various molecular systems, ${ }^{28}$ including porphyrins/conjugated systems. ${ }^{29,30}$ However, TDDFT with standard or even hybrid exchange-correlation functionals breaks down badly in the case of extended systems, such as infinite H-chains and simple polymers. ${ }^{31}$ Recent work towards range-separated exchange functionals resolves many of these problems, although they suffer from several limitations of their own. ${ }^{32-34}$ Quantum chemical techniques, such as $a b$ initio coupled cluster ${ }^{35}$ and configuration interaction $(\mathrm{CI})^{36}$ schemes offer sophisticated treatments of exchange and correlation however with high computational cost (molecules of the size considered in this work are, nonetheless, accessible within the former technique).

An alternative framework for describing excited states is the Green's function approach to many-body perturbation theory (MBPT), ${ }^{37}$ as frequently used in the study of optical spectra of bulk systems, ${ }^{38}$ molecular crystals, ${ }^{39}$ and one-dimensional extended systems such as nanowires, ${ }^{40}$ polymers, ${ }^{41}$ and atomic chains. ${ }^{31}$ Recently, it was demonstrated that the approach yields quantitatively accurate results for electronic ${ }^{42}$ and optical spectra of molecular porphine $\left(\mathrm{H}_{2} \mathrm{P}\right)$ and tetraphenylporphine $\left(\mathrm{H}_{2} \mathrm{TPP}\right){ }^{43}$ Hence the approach should be valid for a consistent study of exciton confinement across the full range of oligomer lengths.

As the oligomers typically synthesized (e.g., octaethylporphyrin dimers) are too large for an ab initio treatment, we consider the smallest possible oligoporphyrin system shown in Fig. 1(a). We correlate the change in polarizability and spectral shifts as a function of the chain length with the redistribution of the excitonic charge density. We demonstrate the

(a)

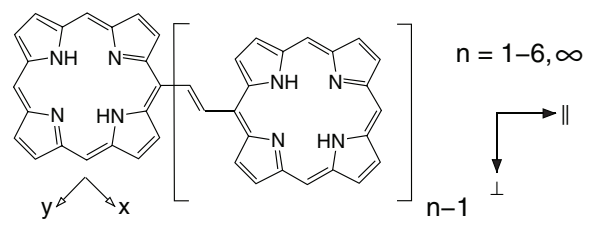

(b)

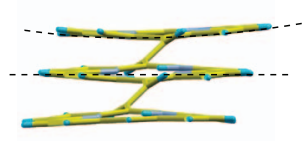

Trimer (c)

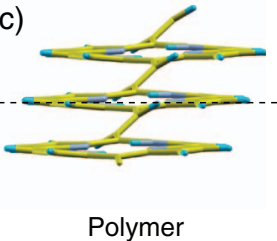

FIG. 1. (a) Molecular structures of porphine oligomers bridged by ethylene $(n=1 \ldots 6, \infty)$. The oligomer axes $(\|, \perp)$ and conventional molecular axes $(x, y)$ of the monomer are shown. (b) End view of trimer showing increased curvature of terminating units. (c) End view of polymer (three units). presence of strongly and weakly bound excitons of WannierMott type, and show that the largest polarizabilities are associated with tightly bound, Frenkel-like excitons. The "four orbital" model of porphyrins is shown to work reasonably well also for the whole suite of porphyrin oligomers. The common practice of extrapolating polymer transition energies from those of oligomers is also analysed from a new perspective.

The paper is structured as follows. Following a brief overview of our theoretical methods, including a critique of the approach (Sec. II), we present a detailed study of the oligomer electronic states from a DFT and MBPT perspective (Sec. III). Optical spectra are presented in Sec. IV along with analyses of the exciton character. An examination of the trends observed with increasing oligomer length and the extrapolation procedure is presented in Sec. V. The paper concludes with some final comments and acknowledgements.

\section{THEORETICAL APPROACH}

\section{A. Formalism}

In this work we employ the so-called $G W$-BSE ( $G W$ approximation-Bethe-Salpeter equation) approach. The formal theory is identical to that described in detail in our previous work on isolated porphine and tetraphenylporphine (Ref. 43), hence it is only briefly described here.

Calculations are performed in three stages. In the first stage, structural relaxations and ground state properties are computed using $\mathrm{DFT}^{44}$ with the Perdew-BurkeErnzenhof (PBE)-generalized gradient approximation (GGA) functional. ${ }^{45}$ At the final geometry a large set of KohnSham (KS) eigenvalues and wavefunctions are then generated within the local-density approximation (LDA). ${ }^{46}$ In the second stage, we obtain the real quasi-particle energies $E_{i}^{Q P}$ as corrections to the KS eigenvalues $E_{i}^{K S}$ through the $G W$ method using the following well known expression from perturbation theory: ${ }^{47}$

$$
\begin{aligned}
E_{i}^{Q P} & =E_{i}^{K S}+\frac{1}{1+\beta_{i}}\left\langle\varphi_{i}^{K S}\left|\Sigma_{x c}\left(E_{i}^{K S}\right)-V_{x c}\right| \varphi_{i}^{K S}\right\rangle \\
& =E_{i}^{K S}+\Delta \Sigma_{G W} .
\end{aligned}
$$

Here $\beta_{i}$ is the linear coefficient in the energy expansion of the self-energy $\Sigma_{x c}$, which is itself the product of the KohnSham Green's function $G$ times the screened Coulomb interaction $W$, obtained within the random phase approximation (RPA). $\Sigma_{x c}$ can be split into two parts, a dynamically screened correlation term $\Sigma_{c}(E)$ and a static exchange term $\Sigma_{x} . V_{x c}$ is the usual DFT exchange-correlation potential. This non-selfconsistent, perturbative $G W$ approach is usually referred to as "single shot" $G_{0} W_{0}$; a popular convention, used later, is to specify the starting wavefunctions explicitly through the form $G_{0} W_{0} @$ LDA.

In the last stage, optical spectra including excitonic and local field effects ( $e-h$ correlation) are computed by means of solving the Bethe-Salpeter equation. By expanding the states over the KS $e-h$ basis, the solution of the BSE can be mapped onto an eigenvalue problem for the excitonic 
Hamiltonian, ${ }^{37,48}$

$$
H_{\mathrm{exc}}=\left(\begin{array}{cc}
R & C \\
-C^{*} & -R^{*}
\end{array}\right),
$$

where the resonant part

$$
R=\left(E_{e}-E_{h}\right) \delta_{e, e^{\prime}} \delta_{h, h^{\prime}}+\left\langle e h|K| e^{\prime} h^{\prime}\right\rangle
$$

is Hermitian, while the coupling part

$$
C=\left\langle e h|K| \overline{e^{\prime} h^{\prime}}\right\rangle
$$

is symmetric. Here $K=W-2 v$ is the excitonic kernel, with $W$ and $v$ being the screened and bare Coulomb interaction, respectively. Electron-hole anti-pairs are denoted by $\overline{e^{\prime} h^{\prime}}{ }^{49}$

A common scheme adopted in computing excited state spectra is the Tamm-Dancoff approximation (TDA), ${ }^{50}$ wherein the coupling terms $C$ are excluded. Within the TDA, the optical response tensor of the supercell (in the limit $\mathbf{q} \rightarrow 0$ ) is computed as

$$
\varepsilon(\omega)=1-v(\mathbf{q}) \sum_{\lambda} \frac{\left|\sum_{v c, \mathbf{k}}\left\langle v, \mathbf{k}+\mathbf{q}\left|e^{-i \mathbf{q} \cdot \mathbf{r}}\right| c, \mathbf{k}\right\rangle A_{\lambda}^{v c, \mathbf{k}}\right|^{2}}{E_{\lambda}-\omega-i \eta}
$$

while the electron-hole wavefunction is obtained from

$$
\chi_{\lambda}\left(r_{e}, r_{h}\right)=\sum_{v c, \mathbf{k}}\left[A_{\lambda}^{v c, \mathbf{k}} \varphi_{c}\left(r_{e}\right) \varphi_{v}^{*}\left(r_{h}\right)\right] .
$$

In the language of MBPT, solving the Bethe-Salpeter equation thus mixes single particle transitions to give the final excitonic spectra, in a similar manner to configuration interaction, with the excitonic eigenfunctions $A_{\lambda}^{c v, \mathbf{k}}$ acting as the mixing coefficients.

Although the TDA works well for typical 3D semiconductor systems, we have shown previously that it fails badly to describe the absorption spectrum of free-base porphyrins ${ }^{43}$ similar effects have been noted for nanotubes ${ }^{49}$ and biomolecules. ${ }^{51}$ Hence we fully account for coupling between resonant and anti-resonant terms in this work, and somewhat more cumbersome equations are needed to describe $\varepsilon$ and $\chi_{\lambda}\left(r_{e}, r_{h}\right)$ : for clarity we report the TDA equations above.

Finally, we extract the polarizability of the isolated molecule or infinite polymer from the dielectric tensor of the repeated supercell using the 3D or 2D Clausius-Mossotti relations. ${ }^{52}$ For the molecular (or oligomer) case, this equates to

$$
\alpha_{\|}=\frac{3 \Omega}{4 \pi} \frac{\epsilon_{\|}-1}{\epsilon_{\|}+2},
$$

with a similar equation for the $\perp$ component. Here $\Omega$ is the supercell volume. For the infinite polymer, the more straightforward expression $\alpha_{\|}=(\Omega / 4 \pi)\left(\epsilon_{\|}-1\right)$ is used for the component of the polarizability along the chain, with $\alpha_{\perp}$ as before. Expressing the optical response in this way allows a direct comparison between intensities of oligomer and polymer spectra to be made.

\section{B. Computational details}

The molecular structure of the species considered in this work is shown in Fig. 1(a). Isolated oligomers and infinite polymer are computed within the same periodic supercell scheme using the quantum-ESPRESSO code ${ }^{53}$ (PWscf version 4.3.1), within a framework of planewaves and normconserving pseudopotentials. Geometries are optimized at the DFT-GGA level (PBE functional ${ }^{45}$ ) at a kinetic energy cutoff of $50 \mathrm{Ry}$, until forces on each atom are less than $1 \mathrm{meV} / \AA$. External symmetries are not imposed. A $\Gamma$-point sampling is used for Brillouin-zone integration, except for the case of the polymer where a $\Gamma$-centred $(8 \times 1 \times 1)$ grid is adopted.

The structure of the free-base monomer is well established, and its symmetry $\left(D_{2 h}\right)$ is correctly found after relaxation. For the dimer, we computed total energies for a number of initial configurations (planar, stepped, twisted) and found that the lowest energy structure consists of coplanar porphyrins (albeit offset vertically by $\sim 1.5 \AA$ ) with the ethylene bridge rotated by $45^{\circ}$ with respect to the macrocyclic planes. These findings agree with those of previous theoretical studies using ethylene bridges. ${ }^{15,16}$ The perfect planarity of the monomer is slightly broken however, and a shallow saddle structure forms in the extremal units, as indicated in Fig. 1(b) for the trimer. In extending to longer oligomers, we assumed a linear configuration with stacking translationally invariant along the chain (U,U conformation), rather than adopt a staggered configuration (U,D form). The polymer is generated using a single monomer unit, plus bridge, periodically repeated, and allowed to relax within a suitable cell (the length is deduced from the inner dimensions of the longer oligomers). By construction, therefore, we have imposed extra symmetry on the polymer with respect to the oligomers (compare Figs. 1(b) and 1(c)). As will become apparent later, this causes imprecision in the extrapolation of trends from oligomer to polymer.

Excited-state properties were computed using the Yambo $\operatorname{code}^{54}$ (version 3.2.5). In order to give a reliable calculation of the trends in the optical absorption for increasing oligomer length, we adopted the same convergence parameters throughout. Due to the computational load, however, we were forced to consider slightly smaller supercells than in our previous work, leading to errors in peak positions of about $0.1 \mathrm{eV}$; furthermore, full $G W$-BSE calculations were only feasible for the three shortest oligomers and polymer. Kohn-Sham states up to $15 \mathrm{eV}$ above the HOMO energy were used in the computation of the quasiparticle corrections in order to keep the band gaps and transition energies converged to below $0.05 \mathrm{eV}$ in each system (absolute energies, such as ionization potentials, are converged to $\sim 0.2 \mathrm{eV}$, however, as a result of this choice). This corresponds to 500 bands (monomer), 1000 bands (dimer), 1500 bands (trimer), and 150 bands (polymer). In each case, a plasmon-pole model was used for the screening; a cutoff of $1600 \mathrm{mHa}$ was used for the dielectric matrix (this corresponds to $9600 \mathrm{G}$-vectors in the trimer). A box-like cutoff in the long-range Coulomb potential is used to simulate truly isolated molecular excited states. This technique is essential $^{55}$ for reaching good convergence (below $0.1 \mathrm{eV}$ ) in the self-energy calculations. For the BSE, we included $e-h$ transitions up to $7.0 \mathrm{eV}$ in the full calculations; as shown later, however, a restricted set of bands is quite adequate for describing the excited state properties of porphyrin systems. Excitonic eigenstates are determined through full diagonalization of the excitonic Hamiltonian, or through Haydock 
inversion using the method of Grüning et al. ${ }^{49}$ Again, $\Gamma$ point sampling is used for the isolated molecules; for the polymer we used up to a $48 \times 1 \times 1 \Gamma$-centred grid in order to plot extended exciton states correctly.

\section{Limitations and approximations}

The physically large size of the supercells, coupled with the high level of theory necessary to obtain quantitatively precise results, means that it is necessary to make various technical approximations or even neglect some phenomena completely. In the former category, dynamical effects in the excitonic screening are neglected in our approach. These are reported to cause a uniform reduction of $0.1 \mathrm{eV}$ in the optical gaps of oligothiophenes. ${ }^{51}$ With regards to physical phenomena, phonon coupling is neglected completely. This effectively means no vibronic replicas appear in our spectra. We also do not consider structural relaxation of excited states, although suitable schemes for $G W$-BSE calculations have been proposed. ${ }^{56}$ Different porphyrin dimers undergo structural relaxation on excitation, to varying degrees. ${ }^{18,26,57}$ For these reasons, we do not attempt a quantitative comparison with experimental spectra, but strive to capture the important physical features and trends in a consistent manner. In any case, comparison with experiment is very much complicated by solvent shifts and solid state effects, ${ }^{58,59}$ or the probable coexistence of different molecular conformers. ${ }^{15,18}$

One possibly serious limitation of our perturbative $G_{0} W_{0}$ approach which has attracted much attention recently (partly due to the increasing application of $G W$-BSE approach to molecular systems) regards the connected topics of (i) dependence of final results on the starting point and (ii) neglect of self-consistency. Recent works have demonstrated that excellent ionization energies and electronic gaps in organic molecules can be obtained using a so-called $G_{0} W_{0} @ \mathrm{HF}_{\text {diag }}$ approach which starts from LDA gaps opened by a Fock-like exchange term; ${ }^{60,61}$ the impact of full self-consistency has also been discussed. ${ }^{62}$

By way of illustration, we show in Fig. 2 the result of carrying out a partially self-consistent $G W_{\mathrm{psc}}$ calculation of the electronic gap in $\mathrm{H}_{2} \mathrm{P}$, whereby eigenvalues are updated

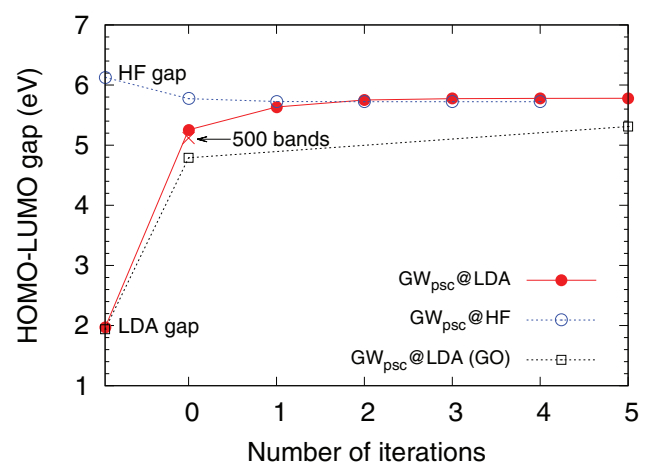

FIG. 2. HOMO-LUMO gap in $\mathrm{H}_{2} \mathrm{P}$, computed within LDA and HartreeFock, and by partially self-consistent $G W_{\mathrm{psc}} @ \mathrm{LDA}$ and $G W_{\mathrm{psc}} @ \mathrm{HF}$ calculations whereby only the eigenvalues are updated. $G W_{\mathrm{psc}} @ \mathrm{LDA}(\mathrm{GO})$ are corresponding data computed using a Gaussian orbital basis, taken from Ref. 60. at each step in both $G$ and $W$. In this way the screening, which depends strongly on the electronic gap, is updated at each step. We consider two very different starting points: DFT-LDA $\left(E_{g}=1.97 \mathrm{eV}\right)$ and pure Hartree-Fock $\left(E_{g}=6.13\right.$ $\mathrm{eV})$, both obtained from fully self-consistent calculations. In the latter case $\left(G W_{\mathrm{psc}} @ \mathrm{HF}\right)$, therefore, only the correlation part of the self energy $\Sigma_{c}$ term is computed at each step, as the exchange term is implicitly included from the beginning. For technical reasons a smaller set of bands is considered here (150 states, i.e., up to $8.5 \mathrm{eV}(10.5 \mathrm{eV})$ above the HOMO when starting from LDA (HF)). The results demonstrate that a self-consistently converged value is reached more quickly when a larger initial gap (due to inclusion of Fock exchange in some form) is considered; in fact, the $G_{0} W_{0} @$ LDA gap underestimates the converged result by about $0.5 \mathrm{eV}$. This is of the same order of the absolute deviation from the experimental ionization potential previously reported within $G_{0} W_{0} @$ LDA. $^{60}$ Proper treatments of these effects are first and foremost beyond the scope of the present work due to computational limitations. Nevertheless, we stress that it is far more important for us to use a consistent and accurate treatment of all systems - from monomer to polymer-in order to quantify the trends correctly, rather than reproduce well one particular experimental result. Moreover, we are more interested in the predictions of the optical spectra from BSE calculations. Screening enters our calculations twice, closing the GW band gap and reducing the exciton binding energy in BSE. As these effects act oppositely in spectral peaks, errors therein tend to cancel out. We recall that self-consistent GW without vertex corrections to the self energy has been a matter of debate for many years. ${ }^{47}$ Indeed, much of the success of the $G_{0} W_{0}$-BSE approach derives from a fortuitous cancellation of errors. In the remainder of this paper, therefore, we neglect the issue of self-consistency, and consider only single shot $G_{0} W_{0} @$ LDA quasiparticle corrections: for brevity this will be referred to simply as "GW."

\section{ELECTRONIC STRUCTURE}

Energy levels calculated within DFT-LDA are plotted in Fig. 3(b) as a function of oligomer length $n$, and isosurface plots $\left(|\psi|^{2}\right)$ of the corresponding orbitals are shown in Fig. 3(a). Energies are shown relative to the vacuum level, as determined from evaluation of the electrostatic potential far from the molecule in each case. Data for the monomer are in agreement with previous calculations at the DFT level. According to its $D_{2 h}$ symmetry, the porphine molecular orbitals (MOs) lying closest to the Fermi level (frontier orbitals) can be grouped into distinct HOMO $\left(b_{1 u}\right)$ and HOMO-1 $\left(a_{u}\right) \pi$ states and a pair of degenerate LUMO $\left(b_{2 g}\right)$ and LUMO+1 $\left(b_{3 g}\right) \pi^{*}$ states. ${ }^{63}$ The orbital ordering for the dimer appears equivalent to that reported in a recent DFT study on a substituted, ethylene-linked $\mathrm{Ni}_{2} \mathrm{P}$ dimer. ${ }^{18}$

The clear energetic separation between the HOMO- and LUMO-derived frontier orbitals and the other states in the system remains true, in fact, for all the systems considered here. These blocks we call "Gouterman" bands, after the eponymous four-orbital model for the porphyrins. ${ }^{64}$ Previously it has been shown how Gouterman's model extends to the 
(a)
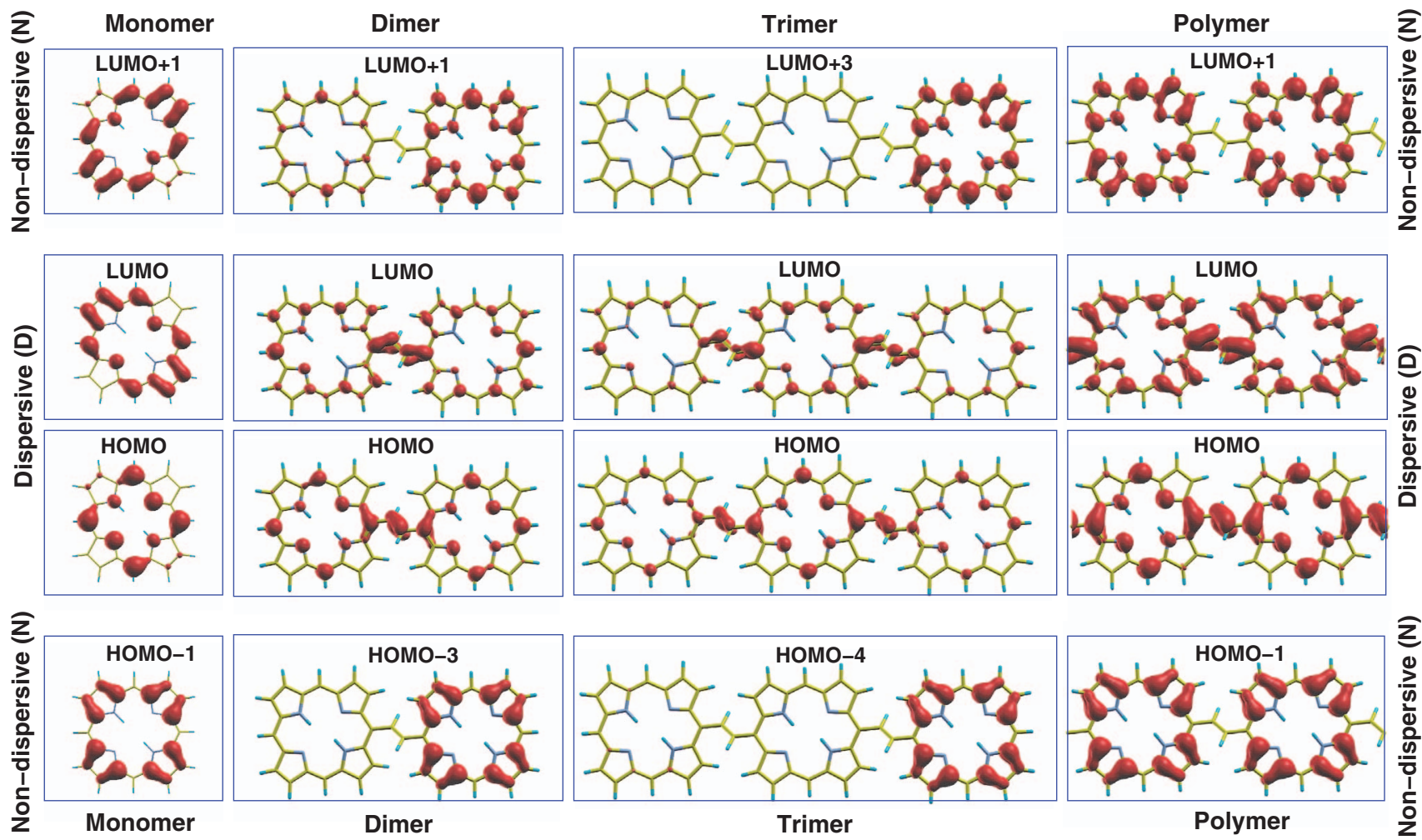

(b)

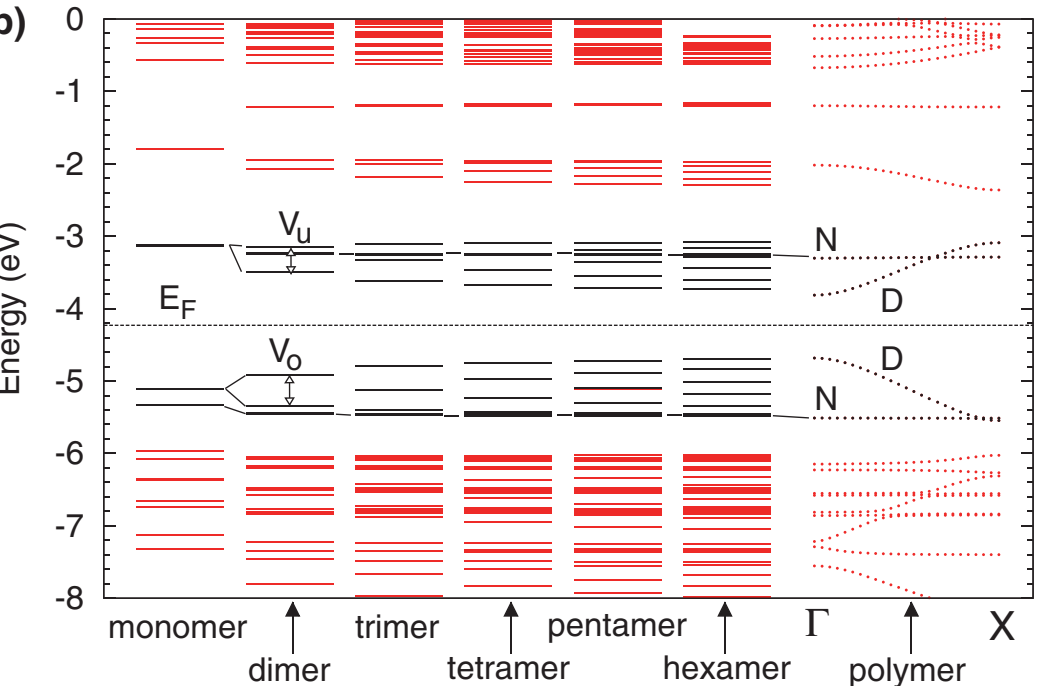

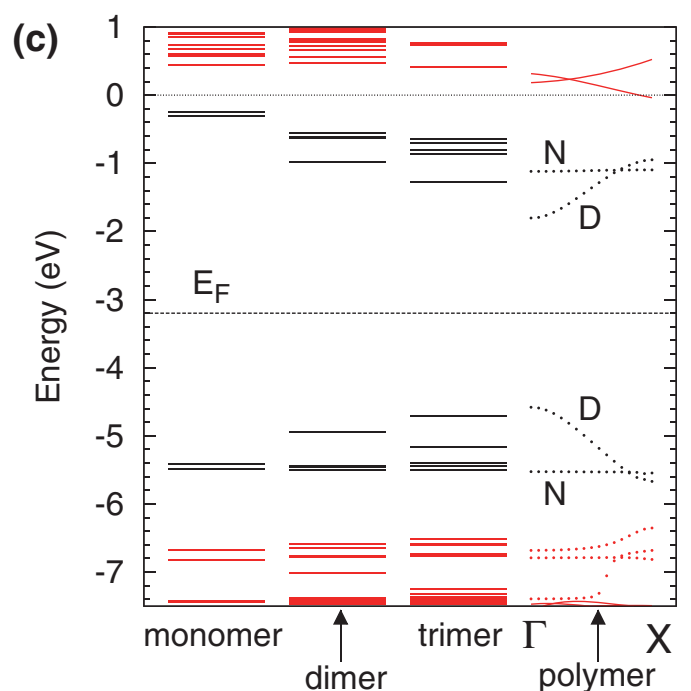

FIG. 3. (a) Isosurface plots of $|\psi|^{2}$ for selected Kohn-Sham orbitals of the porphine oligomers and polymer. Two repeating units are shown for the latter. Rows 2 and 3 show examples of dispersive (D) states; rows 1 and 4 show typical non-dispersive (N) states. The complete sets of frontier orbitals are thus shown for the monomer and polymer only. (b) Kohn-Sham (LDA) eigenvalues, plotted relative to the vacuum level. Black lines and points indicate "Gouterman" levels, which are further characterized as dispersive or non-dispersive bands. (c) as (b), for quasiparticle energies computed using the $G W$ method.

eight-orbital case of the dimer. ${ }^{27,65}$ Each block is composed of "dispersive" $(D)$ orbitals whose energy depends on $n$ and a set of degenerate "non-dispersive" $(N)$ orbitals, as indicated in the figure. The former derive from linear combinations of the $b_{1 u}$ and $b_{2 g}$ MOs in the monomer; these have notable contributions from the $\pi / \pi *$ orbitals of the ethylene bridge, and hence are fully conjugated across the oligomer/polymer length. The monomer HOMO-1 in contrast has a node at the $\mathrm{C}$ atom meso-linked to the bridge, and hence states derived from it remain localized within each macrocyclic unit. A similar separation of localization happens for the LUMO and
LUMO+1 states. (Decomposition into dispersive and nondispersive bands is not restricted to porphyrins; similar analysis has been performed in molecular crystals of $\pi$-bonded sexiphenyl, ${ }^{66}$ for instance.) This consistent orbital character across all oligomers allows a simplified treatment of the optical properties, as discussed later.

As the oligomer length $n$ increases, a number of clear trends become apparent: (i) the energetic positions of both HOMO and LUMO levels slowly converge towards their final values in the infinitely long polymer; (ii) the ionization energy ( $I_{p}$, which correlates with the HOMO energy) decreases with 

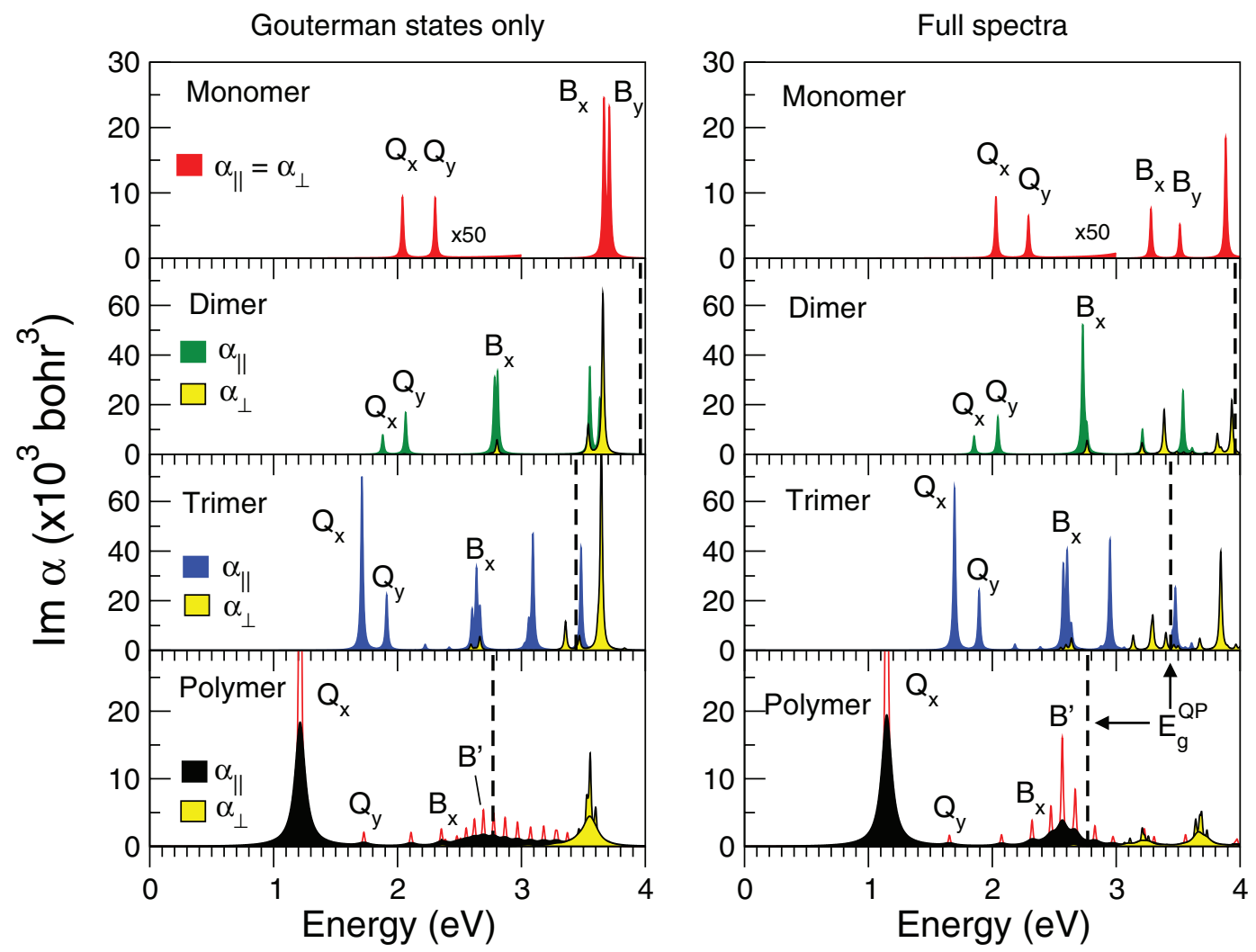

FIG. 4. Absorption spectra of porphyrin oligomers and polymer within $G W$-BSE, for light polarized parallel $\left(\alpha_{\|}\right)$and perpendicular $\left(\alpha_{\perp}\right)$ to the oligomer axis. (Left) Mixing Gouterman (frontier) orbitals only; (right) full spectra. Peak labels $\mathrm{Q}_{x}, \mathrm{Q}_{y}$, etc., are the conventional names for the monomer bands; $x$ and $y$ refer to the axes of the monomer transition dipoles.

increasing $n$; (iii) the KS band gap decreases monotonically towards the polymer gap at $\Gamma$; (iv) the coupling strengths $V_{o}$ and $V_{u}$, i.e., the splitting of the occupied $b_{1 u}$ and unoccupied $b_{2 g}$ orbitals, also decreases monotonically. All four of these trends derive from splitting of states due to the electronic interaction between MOs on adjacent macrocycles: dispersive orbitals form both HOMO and LUMO. The magnitude of the splitting is determined by the coupling strengths $V_{o}$ and $V_{u}$, which depend on the degree of overlap between $\pi$-orbitals as mediated by the ethylene bridge. By comparison, $\beta-\beta$ fused porphyrins yield values of about $1.2 \mathrm{eV}$, far stronger than our case. ${ }^{27}$ It is important to note, however, that the states in the polymer are, by construction, translationally invariant with respect to the unit. In a real (finite) polymer, such states tend to zero near the ends of the polymer chain. ${ }^{67}$

At the quasiparticle level, these four trends still hold. Nevertheless, the improved description of exchange and correlation in the $e-e$ interaction through the electron self-energy $\Sigma$ introduces strong changes, as shown in Fig. 3(c) for the shorter oligomers and polymer. First, the gap considerably opens for all systems. The self-energy correction $\Delta \Sigma_{\mathrm{GW}}$ is largest for the monomer $(3.15 \mathrm{eV})$ where the contribution from $\Sigma_{x}$ is large; moreover, the gap decreases more rapidly with $n$ than the corresponding DFT-LDA gap, until it reaches a value of about $1.90 \mathrm{eV}$ in the polymer. Second, although the Gouterman blocks remain separate from the other orbitals, there is some reordering of levels within each block with respect to the DFT case. Third, $V_{o}$ and $V_{u}$ are increased (similar to the band-stretching reported in $G W$ of semiconductors).
These trends are further reflected by the changes in peak position in the optical absorption spectra for increasing oligomer length, as we will show in Sec. IV.

\section{OPTICAL PROPERTIES}

\section{A. Absorption spectra}

Optical spectra of porphyrins and their derivatives are characterized by two main features: a weak group of peaks, called the $\mathrm{Q}$ bands, in the visible range, and a stronger feature at higher energies termed the $\mathrm{B}$ or Soret band. Their relative intensity and energetic position is explained nicely by the four-orbital model of Gouterman, ${ }^{64}$ whereby single particle transitions between the four frontier MOs (shown in Fig. 3(a) for the monomer) are mixed according to a configuration interaction process. Constructive interference (symmetric combination of transitions) gives rise to the intense $\mathrm{B}$ band, while the weaker Q-band occurs as a result of destructive interference (antisymmetric combination). Due to the inherent $D_{2 h}$ symmetry of free-base porphine, two polarization-dependent $\mathrm{Q}$ bands $\left(\mathrm{Q}_{x}\right.$ and $\left.\mathrm{Q}_{y}\right)$, and two $\mathrm{B}$ bands $\left(\mathrm{B}_{x}\right.$ and $\left.\mathrm{B}_{y}\right)$ are expected in the computed spectrum (the experimental spectrum in particular differs by having distinct vibronic replicas of the $\mathrm{Q}$ bands present). The transition dipole moments are thus oriented along the monomer $(x, y)$ axes (being defined as parallel and perpendicular to the $\mathrm{N}-\mathrm{H} \cdots \mathrm{H}-\mathrm{N}$ bonds: see Fig. 1), which are themselves aligned at about $45^{\circ}$ with respect to the oligomer axes (denoted as $\|, \perp$ ). 
TABLE I. Electronic gaps (DFT-LDA, $G W$ ), $G W$ corrections, spectral peak positions, exciton binding energies (all in eV), and amplitudes $\left(\times 10^{3}\right.$ bohr $\left.^{3}\right)$ for the $\mathrm{Q}_{x}, \mathrm{~B}_{x}$, and $\mathrm{B}^{\prime}$ excitons (full spectra calculation).

\begin{tabular}{|c|c|c|c|c|c|c|c|c|c|c|c|c|}
\hline & \multicolumn{3}{|c|}{ Electronic gap } & \multicolumn{3}{|c|}{ Peak position } & \multicolumn{3}{|c|}{ Binding energy } & \multicolumn{3}{|c|}{ Peak amplitude } \\
\hline & DFT & $G W$ & $\Delta \Sigma_{\mathrm{GW}}$ & $\mathrm{Q}_{x}$ & $\mathrm{~B}_{x}$ & $\mathrm{~B}^{\prime}$ & $\mathrm{Q}_{x}$ & $\mathrm{~B}_{x}$ & $\mathrm{~B}^{\prime}$ & $\mathrm{Q}_{x}$ & $\mathrm{~B}_{x}$ & $\mathrm{~B}^{\prime}$ \\
\hline Monomer & 1.97 & 5.12 & 3.15 & 2.04 & 3.26 & & 3.08 & 1.86 & & 0.2 & 7.6 & \\
\hline Dimer & 1.41 & 3.96 & 2.55 & 1.86 & 2.70 & & 2.10 & 1.26 & & 7.4 & 52.3 & \\
\hline Trimer & 1.18 & 3.44 & 2.25 & 1.67 & 2.55 & & 2.59 & 0.89 & & 65.5 & 41 & \\
\hline Polymer & 0.87 & 2.77 & 1.90 & 1.2 & 2.32 & 2.57 & 1.6 & 0.45 & 0.20 & 97.3 & 3.9 & 16.1 \\
\hline
\end{tabular}

Our previous work ${ }^{43}$ showed that the $G W$-BSE method succeeds in computing reasonable values for the energies and relative intensities of $\mathrm{Q}$ and $\mathrm{B}$ bands in the free-base monomer and tetra-phenyl variant, $\mathrm{H}_{2}$ TPP. In Fig. 4 we show absorption spectra of the monomer, dimer, trimer, and polymer calculated within $G W$-BSE, and summarize the main results in Table I. (Due to computational limitations, it was not possible to compute excitonic spectra for the longer oligomers.) Spectra are shown for polarizations along the two oligomer axes; with the exception of the monomer, the spectra are unsurprisingly dominated by the $\|$-component of the peaks, i.e., along the oligomer chain direction. Vertical dashed lines indicate the $G W$ (electronic) gap, hence all the major peaks correspond to bound excitons, with binding energies, defined here as the difference between the minimum $G W$ gap and the peak energy obtained from BSE, of up to $3 \mathrm{eV}$ (see Table I). A small $(0.01 \mathrm{eV})$ Lorentzian broadening is used for clarity; for the infinite polymer, more broadened spectra $(0.05 \mathrm{eV})$ are also reported (to compensate for the finite $k$-point mesh). Due to the slightly different convergence parameters used, our computed spectrum for the monomer is blueshifted by $\sim 0.05 \mathrm{eV}$ with respect to our previous work; ${ }^{43}$ the agreement with experiment thus remains fair. The $\mathrm{B}_{x}$ peak in the polymer is identified from analysis of the trends in single-particle transitions (mostly D-N transitions, see below). Also indicated is the $\mathrm{B}^{\prime}$ peak, being that having the largest amplitude in the continuum of B bands.

The left column of the figure shows the spectra obtained by mixing only bands from the "Gouterman" blocks identified in Fig. 3(c). With the exception of the monomer, the restriction to the frontier orbitals yields a good description of the optical properties up to the $\mathrm{B}_{x}$ band, especially regarding the peak positions: $\mathrm{Q}$ and $\mathrm{B}_{x}$ bands differ from their converged positions by less than $0.1 \mathrm{eV}$.

As the oligomer length $n$ increases, the absorption spectrum changes in two ways. First, the absolute intensity (see Table I) of the $\mathrm{Q}_{x}$ band increases significantly; the $\mathrm{B}_{x}$ band is largest for the dimer, before falling to a minimum in the infinite polymer case, where it becomes obscured by the stronger $\mathrm{B}^{\prime}$ peak. Indeed, the $\mathrm{Q}_{x}$ amplitude is already greater than that of $\mathrm{B}_{x}$ from the trimer onwards. It is natural to attribute this effect to a larger polarizability of the excitonic wave function in extended molecules, and is discussed in more detail in Sec. IV B. The second obvious change with increasing $n$ is the redshift of both bands (by up to $0.8 \mathrm{eV}$ ) from their corresponding positions in the monomer. Clearly, the peak energies follow the lowering of the electronic (quasiparticle) gaps. However, the peak energies fall faster with $n$ than the gaps do due to the concomitant decrease in the binding energies of both Q and B excitons, reported in Table I. Reduction in binding energy with increasing molecular length has previously been reported in oligoacene crystals. ${ }^{39}$ Changes in the binding energy come from a delicate balance of screened and unscreened exchange-correlation terms in the $e-h$ interaction kernel $K$. As the electronic gap falls with $n$, the direct screened term also decreases, and with it, the $e-h$ binding energy. This acts in the opposite direction to the closing of the $G W$ gap. However, due to the lower $e-h$ confinement in the longer oligomers, the bare exchange term also reduces with $n$. These different contributions, which depend on the nature of the exciton itself, do not exactly cancel, so the overall optical excitation shifts are not as dramatic as those of the electronic gap. The results are in qualitative agreement with available experiments on similar systems. ${ }^{22}$

\section{B. Exciton distribution}

The electron-hole distribution corresponding to the $\mathrm{Q}_{x}$ and $\mathrm{B}_{x}$ excitons, computed for simplicity from the Gouterman spectra, is illustrated in Fig. 5. These plots show the probability of finding an electron while keeping the hole fixed (see Eq. (7)) to a particular macrocyclic unit. In each case, we have taken an average over hole positions that yield the maximum electron amplitude. As expected, these correspond roughly to locations inside the lobes of the HOMO, i.e., about $1 \AA$ above each of the four carbon atoms bridging the pyrrole rings (see Fig. 3(a)). Adding contributions having the hole fixed at the ethylene bridge did not change the distribution function appreciably. The plot is shown both as a $3 \mathrm{D}$ isosurface and as a $1 \mathrm{D}$ planar average along the oligomer axis direction.

The figure demonstrates that there is a clear difference in character between the $\mathrm{Q}_{x}$ and $\mathrm{B}_{x}$ exciton. Both electron and hole of the $\mathrm{Q}_{x}$ exciton are found to be well confined to the same macrocyclic unit, irrespective of oligomer length. This character is most evident in the trimer, where plots are shown restricting the hole to the leftmost and central unit, respectively; these data also indicate that the $\mathrm{Q}$ exciton tends to be localized at the ends of the oligomer (compare the intensities of blue (hole at end) and magenta (hole at centre) curves). The distribution is more spread out in the polymer, most likely due to the perfect symmetry imposed through our calculation method. Nevertheless, the peak in the electron probability always remains in the same macrocycle as the hole. The spatial distribution and binding energy are thus characteristic of a Frenkel exciton; however, the strong electronic coupling between macrocycles, lack of Davydov splitting, and spread of 


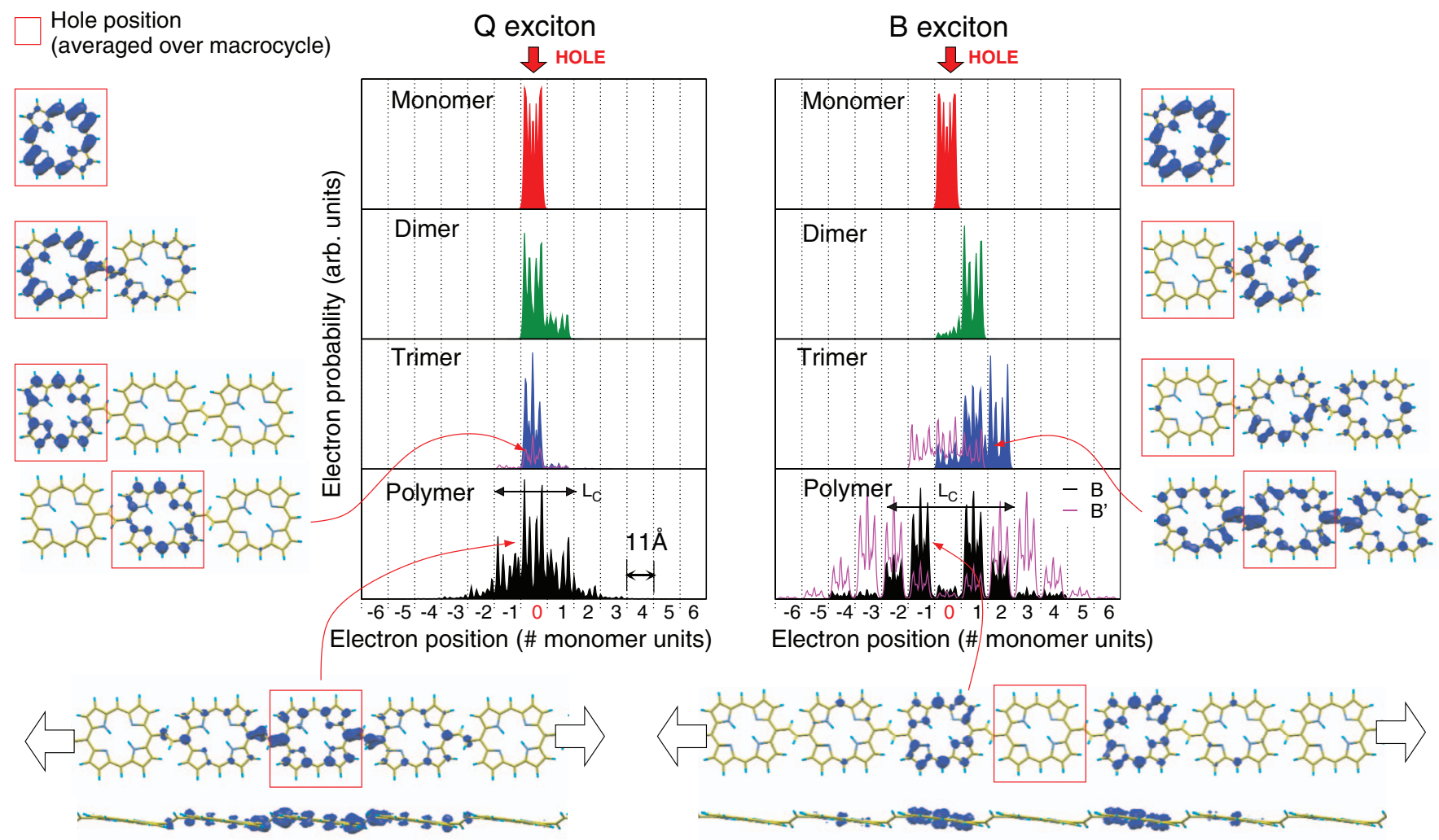

FIG. 5. Exciton distribution in the $\mathrm{Q}_{x}$ and $\mathrm{B}_{x}$ bands for the shortest oligomers and polymer, computed from the Gouterman spectra. Isosurfaces (plotted at one-tenth of the maximum value) are shown at the sides and bottom; planar averaged data are plotted in the centre columns. The hole is confined to within a single macrocyclic unit (see text). For the trimer, two sets of data are shown that correspond to the hole fixed at the end or central unit. $L_{C}$ indicates the exciton coherence length in the polymer for the $\mathrm{Q}_{x}$ and $\mathrm{B}_{x}$ bands.

the excitonic envelope into adjacent units indicates that $\mathrm{Q}_{x}$ has both Frenkel and Wannier-Mott character.

In contrast, the B exciton exhibits a clear WannierMott behaviour, and becomes almost completely chargetransferlike by the polymer. For the latter we show the profiles for the $\mathrm{B}_{x}$ and the very loosely bound $\mathrm{B}^{\prime}$ peaks (magenta line; see also Fig. 4). The coherence length $L_{C}$ (which quantifies the size of the excitonic wavefunction) of the B excitons appears to be limited by the finite size of the oligomers, i.e., the exciton is spread over the whole oligomer length. Hence we cannot estimate directly the delocalization length of the exciton (i.e., where the exciton is likely to be found in the molecule): this quantity can instead be deduced by extrapolation (see Sec. V). The presence of both Frenkellike and Wannier-Mott excitons is a peculiarity of quasi one-dimensional materials. The conjugation along the chain allows a large $e-h$ separation, while the confinement perpendicular to the chain gives rise to the large binding energy.

These results reflect the danger in using polarizability measurements to interpret optical spectra in terms of Frenkel or Wannier excitons in $\pi$-conjugated porphyrin systems. Previously, it has been suggested ${ }^{22}$ that the dramatic increase in intensity of the $\mathrm{Q}$ bands was due to the large polarizability of the exciton (i.e., large dipole moment), which should reflect a weak excitonic binding energy. Our results instead demonstrate that the $\mathrm{Q}$ bands are signatures of small, tightly bound excitons, yet we observe the correct rapid increase in inten- sity. In contrast, although the intensity of the $\mathrm{B}_{x}$ band undergoes more moderate changes, it is clear from our calculations that the B band is indicative of a large, polarizable Wannier or charge transfer exciton.

Such intensity changes have previously been explained on the basis of theoretical calculations on Zn-porphyrin dimers. $^{7,26}$ They ultimately derive from the lowering of molecular symmetry caused by the perturbation of the frontier molecular orbitals through $\pi$-conjugation with the molecular bridge. According to Gouterman's model, the Q band originates from an antisymmetric (destructive) interference of transitions, with an intensity proportional to the difference between the (almost equivalent) dipole moments along the principle axes of the monomer. ${ }^{64}$ As the symmetry lowers, the magnitude and direction of the molecular dipole moments change, and this cancellation is reduced. Our calculations reveal that transitions between dispersive bands (D-to-D) contribute strongest to $\mathrm{Q}_{x}$ : this corresponds to the HOMO-LUMO transition for the extended molecules (occurring near $\Gamma$, for the polymer) and the HOMO to LUMO+1 transition for the monomer (although note that the LUMO and LUMO+1 are practically degenerate in that case). Analysis of the mixing coefficient $A_{\lambda}^{c v, \mathbf{k}}$ (see Eq. (6)) indicates that the net percentage contribution of the HOMO-LUMO transition increases from $3 \%$ in the monomer, to about $37 \%$ (dimer), $75 \%$ (trimer), and finally $>95 \%$ in the polymer. It is this change in the mixing that is ultimately responsible for the dramatic increase in in- 
tensity of the Q band, and not the exciton coherence length which remains fairly constant.

The B peak, which depends more on the sum of the porphyrin dipole moments, is more complicated to analyse as it is composed of different transitions (from the trimer onwards, D-to-N transitions dominate). In this case the increasing $e-h$ distribution also gives rise to a larger dipole moment along the chain. Nonetheless, the contribution of D-type orbitals to the optical spectrum means that the B band certainly undergoes a strong spectral shift. In comparison, butadiyne-linked oligoporphyrins are reported to have a different orbital ordering, and $\mathrm{N}$-to-N transitions dominate the $\mathrm{B}$ band. ${ }^{1}$ In that case, the spectral shift is more modest as only a weaker electrostatic coupling (exciton coupling) is possible between units.

\section{OLIGOMER-POLYMER EXTRAPOLATION: A MBPT PERSPECTIVE}

A large number of studies have already been performed on techniques for extrapolating the optical and electronic properties of conjugated polymers from those of oligomers (for a review, see for instance Gierschner et al. ${ }^{58}$ ). Roughly speaking, the excitation energy of the lowest excited state has a linear relation with respect to the reciprocal of the number of monomer units $(1 / n)$. However, polymer gaps projected by simple linear extrapolations yield underestimated values, and higher order fitting is needed for large $n$ (Refs. 68-70). As discussed by Rissler, ${ }^{71}$ deviations from the linear behaviour can occur for longer oligomers due to extrinsic structural factors such as non-planarity or chain kinking. A first approximation can already be made using the ground state properties of the oligomers, i.e., the bond length alternation (BLA), ${ }^{72}$ defined in the present case as the difference in bond length between the $\mathrm{C}-\mathrm{C}$ bond and adjacent $\mathrm{C}=\mathrm{C}$ bond in the ethylene bridge. The BLA (ca. $0.1 \AA$ for all oligomers studied) indeed decreases with $n$; however, it already saturates at $n=6$. Similar observations were made for instance in oligothiophenes, ${ }^{67}$ in contradiction to the experimental indications that saturation occurs at larger $n{ }^{58}$ As discussed in Ref. 67, this discrepancy is attributed to the shortcomings of ground state approaches.

Important intrinsic factors, deriving from long-range electron correlation phenomena, can also lead to saturation at large $n$. Clearly, the ability to account for intrinsic factors is strongly dependent on the sophistication of the computational framework being used. For time-dependent procedures, it is well known that DFT-based approaches with common functionals (including hybrid functionals like B3LYP) fail to predict the correct trends for increasing molecular length due to an overestimation of the long-range correlation, which leads to an incorrect asymptotic behaviour in the exchange term. ${ }^{29,73}$ As a result, deviation from the linear regime occurs far too late; indeed, DFT/TDDFT studies on oligothiophenes have suggested that more than 20 units $^{70}$ are needed to obtain convergence. Recent work with range-separated density functionals ${ }^{32,33}$ that recover, by design, the correct Fock exchange at large $n$ offer much improved performance. Within quantum-chemical approaches, a description of electron correlation at least at the INDO (intermediate neglect of differential overlap) level ${ }^{74,75}$ is necessary to obtain reason- able trends. ${ }^{26}$ Hartree-Fock CIS agrees well with experiment for geometrically relaxed excited states (adiabatic transitions) with high oscillator strength. ${ }^{76,77}$ Hence it is particularly interesting to look at the performance of the Green's function approaches in predicting polymer transition energies from the oligomer data since in principle they treat correlation effects in an equal manner for both molecules and long or infinite chains.

The first obvious question is how to perform the extrapolation. Several functional forms have been suggested to describe the asymptotic behaviour of the band gap with increasing oligomer length (see Refs. 58-66 of Ref. 58). Here we adopt the physically intuitive approach based on classical mechanics by Kuhn. ${ }^{78}$ In this model, the double bonds of a polyene are regarded as $N$ identical oscillators (of force constant $k_{0}$ ) vibrating at an energy $E_{0}$. If these $N$ oscillators are coupled by a force constant $k$, the resulting lowest energy eigenmode is given by

$$
E_{N}=E_{0} \sqrt{1+2 \frac{k^{\prime}}{k_{0}} \cos \frac{\pi}{N+1}} .
$$

(A similar expression has been derived by Simpson ${ }^{79}$ based on a quantum-mechanical coupling of transition dipole moments associated with the double bonds.) This expression can then be fit to our computed data, taking $E_{0}$ and $k^{\prime} / k_{0}$ as free parameters. The number of double bonds in each oligomer, $N$, is determined by considering the shortest conjugated path connecting the terminal carbon atoms of the molecular backbone. ${ }^{67}$ As illustrated in Fig. 6, there is only one such path of continuous single-double bond alternation possible for the oligomers considered here. As the porphyrin ring structure is very different from that a linear polyene, however, it is interesting to see if such extrapolation can be done for the systems studied here.

Our lowest computed transition energies (electronic and optical gaps) are plotted as a function of $1 / N$ in Fig. 6. Data are shown at various levels of theory: DFT-LDA, $G W$ considering only the exchange term $\Sigma_{x}$ in the self energy, full $G W$ (where possible) and finally $G W$-BSE for the optical gaps. Curved and dashed lines then show fits to these data (using Eq. (9) or a simple linear fit, respectively) for $2 \leq n \leq 6$. Monomers $(n=1)$ were omitted as their bandgaps are significantly lowered due to a different MO topology (no bridge contribution) compared to the longer oligomers, as shown in Fig. 3. Also indicated on the graph is a simple (but robust) graphical method suggested by Gierschner et al. ${ }^{58}$ for extracting a value for the effective conjugation length (ECL), i.e., the point at which the gap saturates with increasing $N$. It is important to stress that the fits do not include the data from the polymer calculations $(1 / N=0)$ : the idea is to compare the polymer data with the values found by extrapolating the oligomer data.

A number of observations can be made. First, and somewhat surprisingly, the data fit Eq. (9) very well. This supports the application of the Kuhn model, designed for linear polyenes, to the more complex macrocyclic structures considered here, so long as the conjugation path is chosen correctly. ${ }^{67}$ 

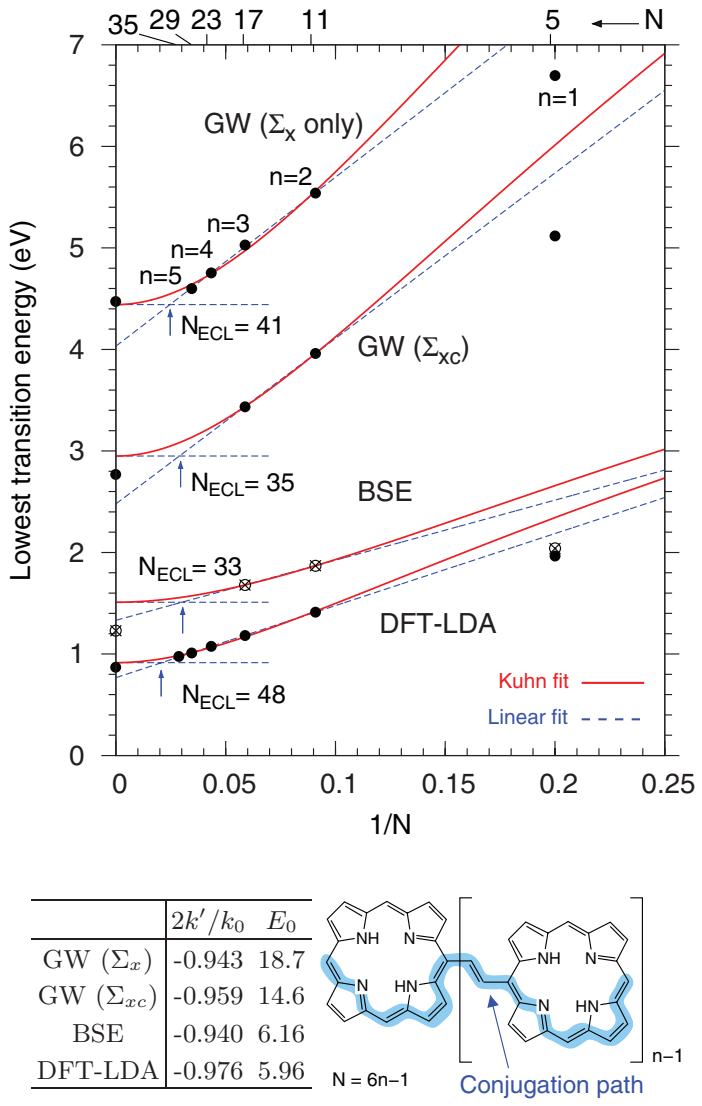

FIG. 6. Lowest transition energy for a range of oligomers $(n=1-6)$ and the infinite polymer, at various levels of theory (LDA, $G W\left(\Sigma_{x}\right.$ only), $G W\left(\Sigma_{\mathrm{xc}}\right)$, and BSE) with respect to $1 / N$, where $N$ is the number of double bonds along the conjugation path (indicated above figure). Linear and Kuhn fits to the data are shown in each case (see text).

Second, large discrepancies (up to $0.3 \mathrm{eV}$ ) between the extrapolated and computed polymer value are evident for full $G W\left(\Sigma_{x c}\right)$ and $G W$-BSE, while for LDA and $\Sigma_{x}$ the agreement is better. The good agreement of LDA may be fortuitous, and simply an artefact of the very small variation in band gap energies from $n=2$ onwards at this level. In contrast, the poor agreement at the higher level of theory points to the incorrect treatment of the polymer structure and symmetry noted earlier: (i) states are, by construction, completely delocalized (translationally invariant) along the chain, whereas oligomers tend to localize the MOs for large $n,{ }^{67}$ and (ii) lowering of the macrocycle symmetry at the end units is not present. Such inconsistency becomes far more apparent when excited states are treated realistically, as they are more sensitive to small deviations.

Third, although the slope for the DFT-LDA calculations is small compared to $G W\left(\Sigma_{x}\right)$ and $G W\left(\Sigma_{x c}\right)$, the $2 k^{\prime} / k_{0}$ parameter, which is the measure for the coupling between the double bonds and thus for delocalization along the backbone, is significantly larger. (The reported values of $2 k^{\prime} / k_{0}$ for $G W\left(\Sigma_{x c}\right)$ and $G W$-BSE are likely to be slightly underestimated due to the availability of only two points in the fitting.) Again, this demonstrates the strong overestimation of delocalization within DFT-LDA.

Fourth, $N_{\mathrm{ECL}}$ varies from $48(n \approx 8)$ within LDA down to 33 ( $n \approx 5-6$ ) estimated with the many body techniques.
The strong difference originates from the different curvature of the polymer extrapolations. High values of $2 k^{\prime} / k_{0}$ and low values of $E_{0}$, as occur for the DFT calculation, lead to flat curvatures with high $N_{\mathrm{ECL}}$. This makes sense, since it shows how effective conjugation is correlated with delocalization.

Returning to the optical gaps, therefore, we obtain an effective conjugation length (33) that is much larger than that of oligothiophenes, for instance $\left(N_{\mathrm{ECL}}=20\right),{ }^{58}$ due to the larger delocalization in the oligo-porphyrins $\left(2 k^{\prime} / k_{0}\right.$ $=0.94$ vs. 0.87 in the thiophenes) and the low monomer bandgap. However, the effective exciton delocalization length in butadiynylene-bridged zinc porphyrin oligomers (a longer bridge than what we consider here) was estimated experimentally to be 3-4 monomer units, ${ }^{22}$ corresponding in that case to $N_{\mathrm{ECL}}=21-28$. This suggests that $N_{\mathrm{ECL}}$ may be roughly the same across different $\pi$-conjugated oligoporphyrins.

The superior description of correlation offered by MBPT thus allows a realistic description of the trends in electronic and optical properties as a function of oligomer length. Careful extrapolation to the infinite limit allows a reasonable value of the exciton delocalization length to be estimated; on the other hand, the limited usefulness of an infinite polymer calculation is clear. A more systematic application of MBPT to simpler $\pi$-conjugated oligomers is therefore most desirable, as we cannot carry out a complete analysis with so few large molecules. Important steps in this direction have been made recently. ${ }^{51}$

\section{CONCLUSIONS}

We have performed DFT and $G W$-BSE calculations of ethylene-linked porphyrin oligomers and the infinite polymer limit. The method is shown to be appropriate for predicting the electronic and optical excited states across the full range of system size. The correct description of the $e-e$ exchange is found to be the crucial quantity in determining the dependence of electronic gap on the oligomer length and in extrapolating to longer molecules. This provides support for the use of range-separated exchange functionals in exploring such systems.

Gouterman's frontier orbital model is shown to give a good description of the full optical absorption spectrum in all systems (at least up to the $\mathrm{B}_{x}$ band energy), with errors in the excitation energies typically less than $0.1 \mathrm{eV}$. In agreement with experimental observations on other $\pi$-conjugated oligoporphyrins, we predict strong redshifts and dramatic increases in the $\mathrm{Q}$ band with increasing oligomer length. Analysis of the excitonic charge distribution reveals that a tightly bound Frenkel-like exciton is responsible for these changes. The higher energy B band is instead explained in terms of Wannier-Mott or charge transfer excitons. The strengths of these shifts are strong indications that these highly conjugated systems lie outside the scope of Kasha's exciton coupling theory model, as noted elsewhere. ${ }^{26}$ We furthermore estimate the delocalization length of the lowest excited state (Q-band) to be of the order of 5 monomer lengths.

Our calculations therefore offer important new insights into interpretation of absorption spectra and polarizability measurements of $\pi$-conjugated oligomers. Strong increases 
in peak intensity are not necessarily derived from extended Wannier-Mott excitons having a large dipole moment, but can instead be derived from excitons confined to one or two monomer units. Further studies are necessary to understand if these effects extend to molecular crystals of porphyrin oligomers. Our findings may also have implications for the use of porphyrin oligomers in photovoltaic applications. Elsewhere it has been suggested that a large intra-molecular delocalization length correlates with a small inter-molecular coupling when the molecules are stacked, which would limit exciton transfer between molecules. ${ }^{80}$ On the other hand, the largest polarizabilities are found to be associated with tightly bound Frenkel-type excitons, which tend not to dissociate easily. Design of an efficient device means harnessing these different characteristics and tuning them in a balanced way.

\section{ACKNOWLEDGMENTS}

The authors are grateful to Dr. Simona Silaghi and Dr. Norbert Esser for useful discussions. Financial support is acknowledged from the European Research Council Advanced Grant DYNamo (ERC-2010-AdG-Proposal No. 267374), Spanish Ministerio de Economia y Competitividad (MINECO, CTQ2011-27317, FIS2011-65702-C02-01, and PIB2010US-00652), ACI-Promociona (ACI2009-1036), Grupos Consolidados UPV/EHU del Gobierno Vasco (IT-31907), and European Commission projects CRONOS (2808792 CRONOS CP-FP7), THEMA (FP7-NMP-2008-SMALL2, 228539) and e-I3 project ETSF-eI3 (INFRA-2007-1.2.2: Grant No. 211956). J.G. is a Ramón y Cajal research fellow of the MINECO. We acknowledge the CINECA Awards SIMPLEX (ISCRA B), 2011 for the availability of high performance computing resources and support, and computational time granted by i2basque, BSC "Red Espanola de Supercomputacion" and SGIker ARINA (UPV/EHU).

${ }^{1}$ D. Kim, Multiporphyrin Arrays: Fundamentals and Applications (Taylor \& Francis, 2012).

${ }^{2}$ J. M. Tour, Chem. Rev. 96, 537 (1996).

${ }^{3}$ J. R. Reimers, T. X. Lü, M. J. Crossley, and N. S. Hush, Nanotechnology 7, 424 (1996).

${ }^{4}$ For a comprehensive list (before 1999), see Refs. 3-14 of H. L. Anderson, Chem. Commun. 1999, 2323.

${ }^{5}$ C. Cutler, A. Burrell, D. Officer, C. Too, and G. Wallace, Synth. Met. 128, 35 (2002).

${ }^{6} \mathrm{~S}$. Shaheen, D. Vangeneugden, R. Kiebooms, D. Vanderzande, T. Fromherz, F. Padinger, C. Brabec, and N. Sariciftci, Synth. Met. 121, 1583 (2001).

${ }^{7}$ M. Drobizhev, Y. Stepanenko, Y. Dzenis, A. Karotki, A. Rebane, P. N. Taylor, and H. L. Anderson, J. Am. Chem. Soc. 126, 15352 (2004); J. Phys. Chem. B 109, 7223 (2005).

${ }^{8}$ M. Senge, M. Fazekas, E. Notaras, W. Blau, M. Zawadzka, O. Locos, and E. NiMhuircheartaigh, Adv. Mater. 19, 2737 (2007).

${ }^{9}$ M. H. Hoang, Y. Kim, M. Kim, K. H. Kim, T. W. Lee, D. N. Nguyen, S.-J. Kim, K. Lee, S. J. Lee, and D. H. Choi, Adv. Mater. 24(39), 5363 (2012).

${ }^{10}$ J. Shinar and R. Shinar, J. Phys. D: Appl. Phys. 41, 133001 (2008).

${ }^{11}$ D. P. Arnold, A. W. Johnson, and M. Mahendran, J. Chem. Soc., Perkin Trans. 1 1978, 366 .

${ }^{12}$ H. L. Anderson, Inorg. Chem. 33, 972 (1994).

${ }^{13}$ V. Lin, S. DiMagno, and M. Therien, Science 264, 1105 (1994).

${ }^{14}$ C. She, S. Easwaramoorthi, P. Kim, S. Hiroto, I. Hisaki, H. Shinokubo, A. Osuka, D. Kim, and J. T. Hupp, J. Phys. Chem. A 114, 3384 (2010).

${ }^{15}$ M. Chachisvilis, V. S. Chirvony, A. M. Shulga, B. Källebring, S. Larsson, and V. Sundström, J. Phys. Chem. 100, 13857 (1996).
${ }^{16}$ M. J. Frampton, H. Akdas, A. R. Cowley, J. E. Rogers, J. E. Slagle, P. A. Fleitz, M. Drobizhev, A. Rebane, and H. L. Anderson, Org. Lett. 7, 5365 (2005).

${ }^{17}$ H. Higuchi, K. Shimizu, Z. Ojima, K.-i. Sugiura, and Y. Sakata, Tetrahedron Lett. 36, 5359 (1995).

${ }^{18}$ O. Locos, B. Bašić, J. C. McMurtrie, P. Jensen, and D. P. Arnold, Chem. Eur. J. 18, 5574 (2012).

${ }^{19}$ M. J. Crossley and P. L. Burn, J. Chem. Soc., Chem. Commun. 1991, 1569.

${ }^{20}$ K. Sendt, L. A. Johnston, W. A. Hough, M. J. Crossley, N. S. Hush, and J. R. Reimers, J. Am. Chem. Soc. 124, 9299 (2002).

${ }^{21}$ M. Kasha, H. Rawls, and M. El-Bayoumi, Pure Appl. Chem. 11, 371 (1965).

${ }^{22}$ J. J. Piet, P. N. Taylor, B. R. Wegewijs, H. L. Anderson, A. Osuka, and J. M. Warman, J. Phys. Chem. B 105, 97 (2001).

${ }^{23}$ W. Barford, R. Bursill, and R. Smith, Phys. Rev. B 66, 115205 (2002).

${ }^{24}$ H. Yamagata and F. C. Spano, J. Chem. Phys. 136, 184901 (2012).

${ }^{25}$ R. Stranger, J. E. McGrady, D. P. Arnold, I. Lane, and G. A. Heath, Inorg. Chem. 35, 7791 (1996).

${ }^{26}$ D. Beljonne, G. E. O'Keefe, P. J. Hamer, R. H. Friend, H. L. Anderson, and J. L. Bredas, J. Chem. Phys. 106, 9439 (1997).

${ }^{27}$ S. Ohira, "Theoretical evaluation of the nonlinear optical properties of extended pi-conjugated chromophores," Ph.D. dissertation (Georgia Institute of Technology, 2009).

${ }^{28}$ See chapters 16-23 in Time-Dependent Density Functional Theory, edited by M. A. L. Marques, C. A. Ullrich, F. Nogueira, A. Rubio, K. Burke, and E. K. U. Gross (Springer, Berlin, 2006) and issue 22 of Phys. Chem. Chem. Phys. 11 (2009).

${ }^{29}$ Z.-L. Cai, K. Sendt, and J. R. Reimers, J. Chem. Phys. 117, 5543 (2002).

${ }^{30}$ E. Baerends, G. Ricciardi, A. Rosa, and S. van Gisbergen, Coord. Chem. Rev. 230, 5 (2002).

${ }^{31}$ D. Varsano, A. Marini, and A. Rubio, Phys. Rev. Lett. 101, 133002 (2008).

${ }^{32}$ L. Kronik, T. Stein, S. Refaely-Abramson, and R. Baer, J. Chem. Theory Comput. 8, 1515 (2012).

${ }^{33}$ U. Salzner, J. Chem. Theory Comput. 7, 2568 (2011).

${ }^{34}$ B. G. Janesko, J. Chem. Phys. 134, 184105 (2011).

${ }^{35}$ See for instance: Recent Progress in Coupled Cluster Methods, edited by P. Cársky, J. Paldus, and J. Pittner (Springer, 2010).

${ }^{36}$ C. D. Sherrill and H. F. Schaefer III, in Advances in Quantum Chemistry, edited by P.-O. Löwdin, J. R. Sabin, M. C. Zerner, and E. Brändas (Academic, 1999), pp. 143-269.

${ }^{37}$ G. Onida, L. Reining, and A. Rubio, Rev. Mod. Phys. 74, 601 (2002).

${ }^{38}$ S. Albrecht, L. Reining, R. Del Sole, and G. Onida, Phys. Rev. Lett. 80, 4510 (1998)

${ }^{39}$ K. Hummer and C. Ambrosch-Draxl, Phys. Rev. B 71, 081202(R) (2005).

${ }^{40}$ M. Bruno, M. Palummo, A. Marini, R. Del Sole, V. Olevano, A. N. Kholod, and S. Ossicini, Phys. Rev. B 72, 153310 (2005).

${ }^{41}$ M. Rohlfing and S. G. Louie, Phys. Rev. Lett. 82, 1959 (1999).

${ }^{42}$ P. Umari, G. Stenuit, and S. Baroni, Phys. Rev. B 79, 201104(R) (2009); 81, 115104 (2010).

${ }^{43}$ M. Palummo, C. Hogan, F. Sottile, P. Bagalá, and A. Rubio, J. Chem. Phys. 131, 084102 (2009).

${ }^{44}$ P. Hohenberg and W. Kohn, Phys. Rev. 136, B864 (1964).

${ }^{45}$ J. Perdew, K. Burke, and M. Ernzerhof, Phys. Rev. Lett. 77, 3865 (1996).

${ }^{46}$ GGA could of course be used throughout; however, GW@GGA calculations are not possible with our code at this time. In any case, the difference in eigenvalues is reported to be small for porphine: see, e.g., Ref. 81 and Fig. 4 of Ref. 43.

${ }^{47}$ F. Aryasetiawan and O. Gunnarsson, Rep. Prog. Phys. 61, 237 (1998); W. G. Aulbur, L. Jönsson, and J. W. Wilkins, Solid State Phys. 54, 1 (1999).

${ }^{48}$ M. Rohlfing and S. Louie, Phys. Rev. B 62, 4927 (2000).

${ }^{49}$ M. Grüning, A. Marini, and X. Gonze, Nano Lett. 9, 2820 (2009).

${ }^{50}$ A. L. Fetter and J. D. Walecka, Quantum Theory of Many-Particle Systems (Dover, New York, 2003), p. 565.

${ }^{51}$ B. Baumeier, D. Andrienko, Y. Ma, and M. Rohlfing, J. Chem. Theory Comput. 8, 997 (2012).

${ }^{52}$ L. Wirtz, M. Lazzeri, F. Mauri, and A. Rubio, Phys. Rev. B 71, 241402(R) (2005).

${ }^{53}$ P. Giannozzi, S. Baroni, N. Bonini, M. Calandra, R. Car, C. Cavazzoni, D. Ceresoli, G. L. Chiarotti, M. Cococcioni, I. Dabo, A. Dal Corso, S. de Gironcoli, S. Fabris, G. Fratesi, R. Gebauer, U. Gerstmann, C. Gougoussis, A. Kokalj, M. Lazzeri, L. Martin-Samos, N. Marzari, F. Mauri, R. Mazzarello, S. Paolini, A. Pasquarello, L. Paulatto, C. Sbraccia, S. Scandolo, G. Sclauzero, A. P. Seitsonen, A. Smogunov, P. Umari, and R. M. Wentzcovitch, J. Phys.: Condens. Matter 21, 395502 (2009). 
${ }^{54}$ A. Marini, C. Hogan, M. Grüning, and D. Varsano, Comput. Phys. Commun. 180, 1392 (2009).

${ }^{55}$ C. Rozzi, D. Varsano, A. Marini, E. Gross, and A. Rubio, Phys. Rev. B 73, 205119 (2006).

${ }^{56}$ S. Ismail-Beigi and S. G. Louie, Phys. Rev. Lett. 90, 076401 (2003).

${ }^{57}$ R. Kumble, G. R. Loppnow, S. Hu, A. Mukherjee, M. A. Thompson, and T. G. Spiro, J. Phys. Chem. 99, 5809 (1995); R. Kumble, S. Palese, V. S.-Y. Lin, M. J. Therien, and R. M. Hochstrasser, J. Am. Chem. Soc. 120, 11489 (1998).

${ }^{58}$ J. Gierschner, J. Cornil, and H.-J. Egelhaaf, Adv. Mater. 19, 173 (2007).

${ }^{59}$ S. Sharifzadeh, A. Biller, L. Kronik, and J. Neaton, Phys. Rev. B 85, 125307 (2012).

${ }^{60}$ X. Blase, C. Attaccalite, and V. Olevano, Phys. Rev. B 83, 115103 (2011).

${ }^{61}$ P. Hahn, W. Schmidt, and F. Bechstedt, Phys. Rev. B 72, 245425 (2005).

${ }^{62}$ F. Caruso, P. Rinke, X. Ren, M. Scheffler, and A. Rubio, Phys. Rev. B 86, 081102(R) (2012).

${ }^{63}$ D. Lamoen and M. Parrinello, Chem. Phys. Lett. 248, 309 (1996).

${ }^{64}$ M. Gouterman, J. Chem. Phys. 30, 1139 (1959).

${ }^{65}$ D. P. Arnold, G. A. Heath, and D. A. James, J. Porphyr. Phthalocyanines 3, 5 (1999).

${ }^{66}$ G. Koller, S. Berkebile, M. Oehzelt, P. Puschnig, C. Ambrosch-Draxl, F. P. Netzer, and M. G. Ramsey, Science 317, 351 (2007).

${ }^{67}$ B. Milián-Medina and J. Gierschner, WIREs Comput. Mol. Sci. 2, 513 (2012).
${ }^{68}$ G. Hutchison, Y.-J. Zhao, B. Delley, A. Freeman, M. Ratner, and T. Marks, Phys. Rev. B 68, 035204 (2003).

${ }^{69}$ S. S. Zade, N. Zamoshchik, and M. Bendikov, Acc. Chem. Res. 44, 14 (2011).

${ }^{70}$ S. S. Zade and M. Bendikov, Org. Lett. 8, 5243 (2006).

${ }^{71}$ J. Rissler, Chem. Phys. Lett. 395, 92 (2004).

${ }^{72}$ M. Kertesz, Chem. Phys. 44, 349 (1979); B. M. Wong and J. G. Cordaro, J. Phys. Chem. C 115, 18333 (2011).

${ }^{73}$ J. R. Reimers, Z.-L. Cai, A. Bilić, and N. S. Hush, Ann. N. Y. Acad. Sci. 1006, 235 (2003).

${ }^{74}$ M. C. Zerner, in Reviews in Computational Chemistry, edited by K. B. Lipkowitz, and D. B. Boyd (VCH, New York, 1991), pp. 313-365.

${ }^{75}$ M. Kotzian, N. Rösch, and M. C. Zerner, Theor. Chim. Acta 81, 201 (1992).

${ }^{76}$ J. Gierschner, H.-G. Mack, L. Luer, and D. Oelkrug, J. Chem. Phys. 116, 8596 (2002).

${ }^{77}$ J. Gierschner, H.-G. Mack, H.-J. Egelhaaf, S. Schweizer, B. Doser, and D. Oelkrug, Synth. Met. 138, 311 (2003).

${ }^{78}$ W. Kuhn, Helv. Chim. Acta 31, 1780 (1948).

${ }^{79}$ T. Simpson and O. F. Washington, J. Am. Chem. Soc. 77, 6164 (1955).

${ }^{80}$ J. Gierschner, Y.-S. Huang, B. Van Averbeke, J. Cornil, R. H. Friend, and D. Beljonne, J. Chem. Phys. 130, 044105 (2009).

${ }^{81}$ A. Ghosh and T. Vangberg, Theor. Chim. Acta 97, 143 (1997). 